\title{
Platinum-Catalyzed Intermolecular Hydroamination of Unactivated Olefins with Carboxamides
}

Xiang Wang and Ross A. Widenhoefer*

P. M. Gross Chemical Laboratory

Duke University

Durham, NC 27708-0346

email: rwidenho@chem.duke.edu

\section{Supporting Information}

Experimental procedures and spectroscopic data for alkylated carboxamides and 3-ethyl-2oxazolidinone (4 pages). 


\section{Experimental}

General Methods. Pressurized reactions were performed behind a 3/8 inch plexiglass blast shield in a thick-walled glass tube equipped with a pressure gauge and a valve that allowed the pressure of the tube to be controlled throughout the transformation. NMR spectra were obtained on a Varian spectrometer operating at $400 \mathrm{MHz}$ for ${ }^{1} \mathrm{H}$ NMR and $100 \mathrm{MHz}$ for ${ }^{13} \mathrm{C}$ NMR in $\mathrm{CDCl}_{3}$ unless otherwise noted. Gas chromatography was performed on a Hewlett-Parkard 5890 gas chromatography equipped with a $25 \mathrm{~m}$ polydimethylsiloxane capillary column. Flash column chromatography was performed employing 200-400 mesh silica gel (EM). Thin layer chromatography (TLC) was performed on silica gel $60 \mathrm{~F}_{254}$ eluting with a 5:1 mixture of hexanes and ethyl acetate unless otherwise noted. 1,4-Dioxane (Acros, anhydrous), $\left[\mathrm{PtCl}_{2}\left(\mathrm{H}_{2} \mathrm{C}=\mathrm{CH}_{2}\right)\right]_{2}$ (1) (Strem), phosphines, carboxamides, and 2-oxazolidone (Aldrich) were used as received.

$N$-Ethylbenzamide (Table 1, entry 9). A solution of benzamide (121 mg, $1.0 \mathrm{mmol}), 1$ (15 mg, $\left.2.5 \times 10^{-3} \mathrm{mmol}\right), \mathrm{PPh}_{3}(13 \mathrm{mg}, 0.05 \mathrm{mmol})$ in dioxane $(0.5 \mathrm{~mL})$ in a thick-walled tube was evacuated, pressurized with ethylene (50 psi), and heated to $120^{\circ} \mathrm{C}$ while maintaining constant pressure. The reaction mixture was heated at $120^{\circ} \mathrm{C}$ for $24 \mathrm{~h}$, cooled to room temperature, and chromatographed $\left(\mathrm{SiO}_{2}\right.$, hexanes-EtOAc $\left.=2: 1\right)$ to give $N$-ethylbenzamide $(144 \mathrm{mg}, 97 \%)$ as a pale yellow solid. mp 71-72 ${ }^{\circ} \mathrm{C}$. ${ }^{1} \mathrm{H}$ NMR $\delta$ 7.74-7.77 (m, $\left.2 \mathrm{H}\right), 7.44-7.48$ (m, $\left.1 \mathrm{H}\right)$, 7.37-7.41 (m, 2 $\mathrm{H}), 6.41$ (br s, $1 \mathrm{H}), 3.46(\mathrm{dq}, J=5.6,7.2 \mathrm{~Hz}, 2 \mathrm{H}), 1.22(\mathrm{t}, J=7.2 \mathrm{~Hz}, 3 \mathrm{H}) .{ }^{13} \mathrm{C}\left\{{ }^{1} \mathrm{H}\right\} \mathrm{NMR}: \delta$ $167.7,135.0,131.5,128.7,127.1,35.1,15.1$.

$N$-Ethyl-4-methoxybenzamide (Table 2, entry 1). mp $76-77{ }^{\circ} \mathrm{C} .{ }^{1} \mathrm{H}$ NMR: $\delta 7.73$ $(\mathrm{d}, J=8.8 \mathrm{~Hz}, 2 \mathrm{H}), 6.81(\mathrm{~d}, J=8.8 \mathrm{~Hz}, 2 \mathrm{H}), 3.75(\mathrm{~s}, 3 \mathrm{H}), 3.39(\mathrm{dq}, J=6.0,7.2 \mathrm{~Hz}, 2 \mathrm{H}), 1.15$ (t, $J=7.2 \mathrm{~Hz}, 3 \mathrm{H}) .{ }^{13} \mathrm{C}\left\{{ }^{1} \mathrm{H}\right\} \mathrm{NMR}: \delta 167.4,162.1,129.0,127.2,113.7,55.5,35.0,15.1$.

$N$-Ethyl-4-bromobenzamide (Table 2, entry 2). mp $110-111^{\circ} \mathrm{C} .{ }^{1} \mathrm{H}$ NMR: $\delta 7.61$ $(\mathrm{d}, J=8.8 \mathrm{~Hz}, 2 \mathrm{H}), 7.28(\mathrm{~d}, J=8.4 \mathrm{~Hz}, 2 \mathrm{H}), 6.78(\mathrm{br} \mathrm{s}, 1 \mathrm{H}), 3.42(\mathrm{dq}, J=5.6,7.2 \mathrm{~Hz}, 2 \mathrm{H}), 1.19$ $(\mathrm{t}, J=7.2 \mathrm{~Hz}, 3 \mathrm{H}) .{ }^{13} \mathrm{C}\left\{{ }^{1} \mathrm{H}\right\} \mathrm{NMR}: \delta 166.8,133.8,131.8,128.8,35.2,15.0$. 
$N$-Ethyl-4-nitrobenzamide (Table 2, entry 3). mp 140-142 ${ }^{\circ} \mathrm{C} .{ }^{1} \mathrm{H}$ NMR: $\delta 8.22(\mathrm{~d}$, $J=8.4 \mathrm{~Hz}, 2 \mathrm{H}), 7.92(\mathrm{~d}, J=8.4 \mathrm{~Hz}, 2 \mathrm{H}), 6.70(\mathrm{~s}, 1 \mathrm{H}), 3.45-3.51(\mathrm{~m}, 2 \mathrm{H}), 1.24(\mathrm{t}, J=7.2 \mathrm{~Hz}, 3$ H). ${ }^{13} \mathrm{C}\left\{{ }^{1} \mathrm{H}\right\} \mathrm{NMR}: \delta$ 165.7, 149.6, 140.6, 128.3, 123.9, 35.5, 14.9.

$N$-Ethyl-4-toluamide (Table 2, entry 4). ${ }^{1} \mathrm{H}$ NMR: $\delta 7.67(\mathrm{~d}, J=8.4 \mathrm{~Hz}, 2 \mathrm{H}), 7.11$ $(\mathrm{d}, J=8.0 \mathrm{~Hz}, 2 \mathrm{H}), 6.94(\mathrm{~s}, 1 \mathrm{H}), 3.39$ (dq, $J=6.0,7.2 \mathrm{~Hz}, 2 \mathrm{H}), 2.31$ (s, $3 \mathrm{H}), 1.15$ (t, $J=7.2 \mathrm{~Hz}$, $3 \mathrm{H}) .{ }^{13} \mathrm{C}\left\{{ }^{1} \mathrm{H}\right\} \mathrm{NMR}: \delta 167.8,141.6,132.1,129.2,127.2,35.0,21.6,15.0$.

$\boldsymbol{N}$-Ethyl-2-toluamide (Table 2, entry 5). mp $48-49{ }^{\circ} \mathrm{C} .{ }^{1} \mathrm{H}$ NMR: $\delta$ 7.20-7.24 (m, 2 H), 7.07-7.13 (m, 2 H), 6.20 (m, 1 H), 3.33 (dq, $J=6.0,7.2 \mathrm{~Hz}, 2 \mathrm{H}), 2.34$ (s, $3 \mathrm{H}), 1.14$ (t, $J=7.2$ $\mathrm{Hz}, 3 \mathrm{H}) .{ }^{13} \mathrm{C}\left\{{ }^{1} \mathrm{H}\right\}$ NMR: $\delta$ 170.3, 136.9, 135.9, 131.0, 129.7, 126.8, 125.8, 34.8, 19.8, 15.0 .

$N$-Ethyl-2-naphthylcarboxamide (Table 2, entry 6). mp $125-126{ }^{\circ} \mathrm{C} .{ }^{1} \mathrm{H}$ NMR: $\delta$ 8.27 (s, 1 H), 7.79-7.84 (m, 4 H), 7.74-7.53 (m, 2 H), 6.74 (br s, 1 H), 3.52 (dq, J = 5.6, 7.2 Hz, 2 $\mathrm{H}), 1.25(\mathrm{t}, J=7.2 \mathrm{~Hz}, 3 \mathrm{H}) .{ }^{13} \mathrm{C}\left\{{ }^{1} \mathrm{H}\right\} \mathrm{NMR}: \delta 167.8,134.8,132.8,132.2,129.1,128.5,127.9$, $12.7,127.6,126.8,123.9,35.3,15.1$.

$N$-Ethyl-1-naphthylcarboxamide (Table 2, entry 7). mp 101-102 ${ }^{\circ} \mathrm{C} .{ }^{1} \mathrm{H}$ NMR: $\delta$ 8.21- 8.23 (m, $1 \mathrm{H}), 7.79-7.83(\mathrm{~m}, 2 \mathrm{H}), 7.44-7.50(\mathrm{~m}, 3 \mathrm{H}), 7.32(\mathrm{dd}, J=7.2,8.4 \mathrm{~Hz}, 1 \mathrm{H}), 6.33$ (br s, $1 \mathrm{H}), 3.43(\mathrm{dq}, J=6.0,7.2 \mathrm{~Hz}, 2 \mathrm{H}), 1.18(\mathrm{t}, J=7.2 \mathrm{~Hz}, 2 \mathrm{H}) .{ }^{13} \mathrm{C}\left\{{ }^{1} \mathrm{H}\right\} \mathrm{NMR}: \delta 169.7$, $134.9,133.8,130.5,130.3,128.4,127.1,126.5,125.6,125.6,125.0,124.9,35.0,15.0$.

$N$-Ethylvaleramide (Table 2, entry 8). ${ }^{1} \mathrm{H}$ NMR: $\delta 6.28(\mathrm{br} \mathrm{s}, 1 \mathrm{H}), 3.18(\mathrm{dq}, J=5.6$, $7.2 \mathrm{~Hz}, 2 \mathrm{H}), 2.08$ (t, $J=7.6 \mathrm{~Hz}, 2 \mathrm{H}), 1.48-1.55(\mathrm{~m}, 2 \mathrm{H}), 1.25$ (sept, $J=7.6 \mathrm{~Hz}, 2 \mathrm{H}), 1.04$ (t, $J=$ $7.2 \mathrm{~Hz}, 3 \mathrm{H}), 0.82(\mathrm{t}, J=7.2 \mathrm{~Hz}, 3 \mathrm{H}) .{ }^{13} \mathrm{C}\left\{{ }^{1} \mathrm{H}\right\} \mathrm{NMR}: \delta$ 173.5, 36.6, 34.3, 28.1, 22.5, 14.9, 13.9.

$N$-Ethylcyclohexanecarboxamide (Table 2, entry 9). mp 92-93 ${ }^{\circ} \mathrm{C} .{ }^{1} \mathrm{H}$ NMR; $\delta$ $5.63(\mathrm{br} \mathrm{s}, 1 \mathrm{H}), 3.24(\mathrm{dq}, J=5.6,7.2 \mathrm{~Hz}, 2 \mathrm{H}), 2.02(\mathrm{tt}, J=7.2,15.0 \mathrm{~Hz}, 2 \mathrm{H}), 1.73-1.83(\mathrm{~m}, 4 \mathrm{H})$, 1.62-1.66 (m, $1 \mathrm{H}), 1.36-1.47(\mathrm{~m}, 2 \mathrm{H}), 1.14-1.28(\mathrm{~m}, 3 \mathrm{H}), 1.09(\mathrm{t}, J=7.2 \mathrm{~Hz}, 3 \mathrm{H}) .{ }^{13} \mathrm{C}\left\{{ }^{1} \mathrm{H}\right\}$ NMR: $\delta$ 176.2, 45.7, 34.3, 29.9, 25.9, 15.1 . 
$N$-Ethylpivalamide (Table 2, entry 10). mp $44-45{ }^{\circ} \mathrm{C} .{ }^{1} \mathrm{H}$ NMR: $\delta 5.75(\mathrm{br} \mathrm{s}, 1 \mathrm{H})$, $3.20(\mathrm{dq}, J=5.6,7.2 \mathrm{~Hz}, 2 \mathrm{H}), 1.13(\mathrm{~s}, 9 \mathrm{H}), 1.06(\mathrm{t}, J=7.2 \mathrm{~Hz}, 3 \mathrm{H}) .{ }^{13} \mathrm{C}\left\{{ }^{1} \mathrm{H}\right\} \mathrm{NMR}: \delta 178.5$, 38.7, 34.5, 27.7, 15.0.

$N$-Ethyl-2-piperidinone (Table 2, entry 11). ${ }^{1} \mathrm{H}$ NMR: $\delta 3.25$ (q, $\left.J=7.2 \mathrm{~Hz}, 2 \mathrm{H}\right)$, 3.11-3.14 (m, $2 \mathrm{H}), 2.19$ (t, $J=6.4 \mathrm{~Hz}, 2 \mathrm{H}), 1.61-1.67(\mathrm{~m}, 2 \mathrm{H}), 0.96(\mathrm{t}, J=7.2 \mathrm{~Hz}, 3 \mathrm{H}) .{ }^{13} \mathrm{C}$ $\left\{{ }^{1} \mathrm{H}\right\}$ NMR: $\delta$ 169.3, 47.2, 41.8, 32.4, 23.3, 21.5, 12.2.

3-Ethyl-2-oxazolidinone (Table 2, entry 12). ${ }^{1} \mathrm{H}$ NMR: $\delta$ 4.29-4.33 (m, $2 \mathrm{H}$ ), 3.52$3.57(\mathrm{~m}, 2 \mathrm{H}), 3.32(\mathrm{q}, J=7.2 \mathrm{~Hz}, 2 \mathrm{H}), 1.16(\mathrm{q}, J=7.2 \mathrm{~Hz}, 3 \mathrm{H}) .{ }^{13} \mathrm{C}\left\{{ }^{1} \mathrm{H}\right\} \mathrm{NMR}: \delta 158.4$, 61.9, 44.0, 39.0, 12.6 .

$N$-Isopropylvaleramide (eq 2). A thick-walled tube containing valeramide (101 mg, 1.0 $\mathrm{mmol}), 1$ (30 mg, $0.05 \mathrm{mmol}), \mathrm{PPh}_{3}(26 \mathrm{mg}, 0.10 \mathrm{mmol})$ and dioxane $(0.5 \mathrm{~mL})$ was evacuated, pressurized with propylene (100 psi), and heated to $120^{\circ} \mathrm{C}$ while maintaining constant pressure. The reaction mixture was heated at $120{ }^{\circ} \mathrm{C}$ for $80 \mathrm{~h}$, cooled to room temperature and chromatographed $\left(\mathrm{SiO}_{2}\right.$, hexanes-EtOAc $\left.=2: 1\right)$ to give $N$-isopropylvaleramide $(105 \mathrm{mg}, 73 \%)$ as a pale yellow oil. ${ }^{1} \mathrm{H}$ NMR $\delta 5.44(\mathrm{br} \mathrm{s}, 1 \mathrm{H}), 4.05$ (d sept, $\left.J=5.2,10.8 \mathrm{~Hz}, 1 \mathrm{H}\right), 2.10(\mathrm{t}, J=7.6$ Hz, 2 H), 1.53-1.61 (m, 2 H), 1.31 (sextet, $J=7.2 \mathrm{~Hz}, 2 \mathrm{H}), 1.11$ (d, $J=6.4 \mathrm{~Hz}, 6 \mathrm{H}), 0.88$ (t, $J=$ $7.2 \mathrm{~Hz}, 3 \mathrm{H}) .{ }^{13} \mathrm{C}\left\{{ }^{1} \mathrm{H}\right\}$ NMR: $\delta$ 172.5, 41.4, 36.9, 28.1, 23.0, 25.6, 14.0. 